\title{
Spin polarization of Bloch states and Hall currents in GaAs quantum wells
}

\author{
Pavel Středa ${ }^{\mathrm{a}}$ and Václav Drchal ${ }^{\mathrm{b}}$ \\ Institute of Physics ASCR, Na Slovance 2, 18221 Praha 8, Czech Republic
}

\begin{abstract}
Real-space distribution of the Hall current densities and their spin polarization in non-magnetic zinc-blende semiconductors is analysed. It is shown that the applied current gives rise to a local spin polarization accompanied by spin-polarized Hall-current densities. Especially for hole carriers they are responsible for transferring spins of different orientation in opposite directions, spin Hall current, while the total Hall current vanishes.
\end{abstract}

\section{Introduction}

The description of the carrier properties in zinc-blende semiconductors is usually based on the Kane or Luttinger models [1] which capture most of the semiconductor properties. A key ingredient of these models is the spin-orbit interaction which couples the momentum with the orbital and spin degrees of freedom giving rise to a spin splitting in the k-space. Energy spectrum and spin orientation of carriers are established by the use of the effective medium Hamiltonian [2] which depends on the wave vector $\mathbf{k}$. In the case of the equilibrium occupation of states the total spin vanishes at any point in real space, which is the consequence of the Kramer's theorem. However, the nonequilibrium occupation induced by the applied current leads to the spin polarization of the system [3-5]. As predicted by the spin-Hall-effect theory, [6-9] it should be accompanied by the spin-Hall current. The spin current operator is usually defined as a symmetrized product of the spin density and velocity. Unfortunately, this definition has no obvious physical meaning $[10,11]$.

In this paper the real-space spin distribution in nonmagnetic zinc-blende semiconductors will be analysed to establish the spatial distribution of the Hall current densities and their spin polarization. It will be shown that particularly for $p$-band states they are able to transfer spins of different orientation in opposite directions while the total Hall current vanishes.

Real space analysis requires the knowledge of Bloch eigenfunctions which excludes the use of the effective medium approach. We have used empirical pseudopotential method with parameters representing GaAs crystals for their evaluation $[12,13]$. Obtained energy dispersions as well spin polarization of Bloch states show the same features as those given by the effective medium approach.

We limit our attention to a two-dimensional carrier gas confined along the $[0,0,1]$ crystallographic direction. Its width standardly exceeds ten lattice constants. It suggests that in this case the carrier properties could be approximated as properties of carriers in the bulk crystal with

\footnotetext{
a e-mail: streda@fzu.cz

b The authors acknowledge support from the Czech Science Foundation, Project P204/11/1228.
}

vanishing $k$-vector component perpendicular to the carrier layer, $k_{z}=0$. This approximation ignoring real conditions at the potential well boundaries can thus be applicable to a two-dimensional system realized in wide enough wells.

It can be expected that the current-induced real-space distribution of the Hall current densities and their spin polarization depends on the orbital momentum of carriers. For this reason the numerical results will be presented for s-band electrons having nearly zero orbital momentum and light holes as an example of carriers having the p-type character with non-zero momentum.

\section{Model Hamiltonian}

Standard single-electron Hamiltonian including spin-orbit coupling is of the following form

$$
H=\left[\frac{p^{2}}{2 m_{0}}+V(\mathbf{r})\right] \sigma_{0}+\gamma \sigma \cdot[\nabla V(\mathbf{r}) \times \mathbf{p}],
$$

where $\gamma$ denotes spin-orbit coupling constant $\gamma=\lambda_{c}^{2} / 4 \hbar$ with Compton length $\lambda_{c}=\hbar /\left(m_{0} c\right)=2.43 \cdot 10^{-12} \mathrm{~m}$. Components of the spin operator $\sigma$ are Pauli matrices

$$
\sigma_{x} \equiv\left(\begin{array}{cc}
0 & 1 \\
1 & 0
\end{array}\right), \sigma_{y} \equiv\left(\begin{array}{cc}
0 & -i \\
i & 0
\end{array}\right), \sigma_{z} \equiv\left(\begin{array}{cc}
1 & 0 \\
0 & -1
\end{array}\right),
$$

and $\sigma_{0}$ stands for a unit matrix. Translation symmetry of the crystalline potential $V(\mathbf{r})$ implies that eigenfunctions are of the Bloch form

$$
\begin{array}{r}
\langle\mathbf{r} \mid n, s, \mathbf{k}\rangle=\Psi_{n, s, \mathbf{k}}(\mathbf{r})=\frac{1}{\sqrt{8 \pi^{3}}} e^{i \mathbf{k r}} u_{n, s}(\mathbf{k}, \mathbf{r}), \\
u_{n, s}\left(\mathbf{k}, \mathbf{r}+\mathbf{R}_{i}\right)=u_{n, s}(\mathbf{k}, \mathbf{r}),
\end{array}
$$

where $\mathbf{R}_{i}$ is the position vector of the $i$-th Wigner-Seitz cell and $(n, s)$ denote energy bands. Periodic parts of Bloch functions $u_{n, s}(\mathbf{k}, \mathbf{r})$ are spinors which can be expressed as a Fourier series

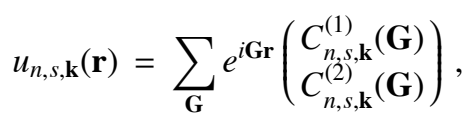

where $\mathbf{G}$ are reciprocal lattice vectors. 


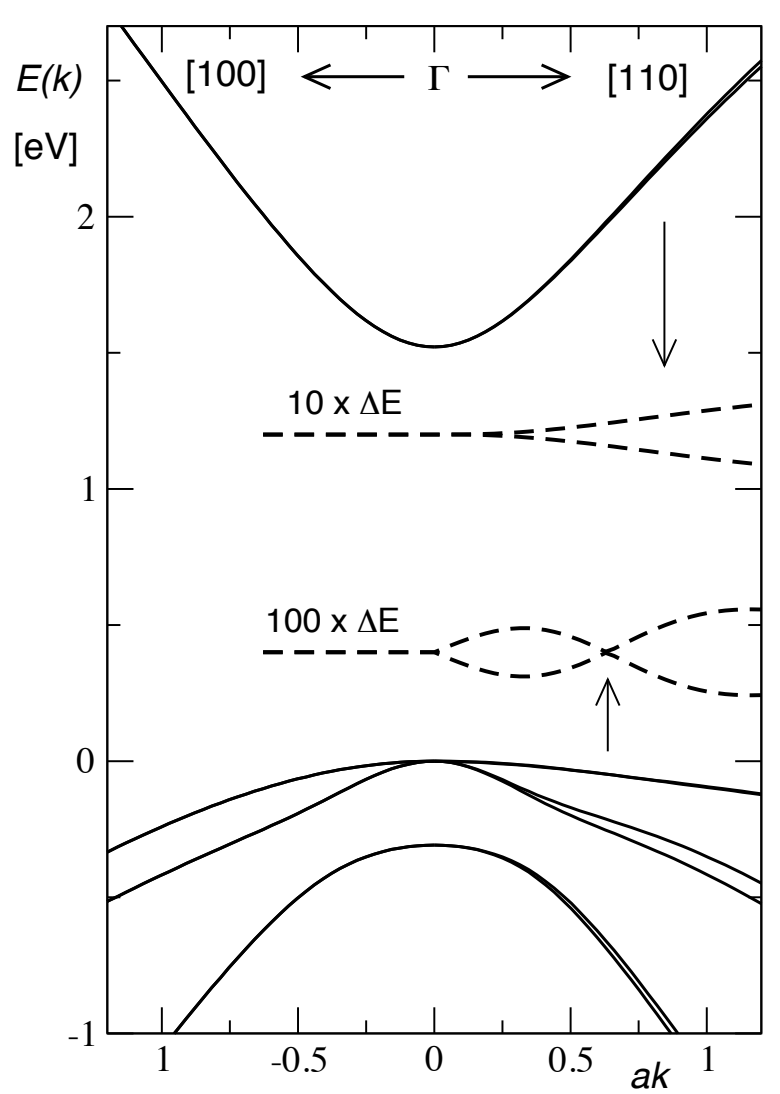

Fig. 1. Energy dispersion laws of GaAs s- and p-bands in the vicinity of the $\Gamma$-point along the [100] and [110] directions for $k_{z}=0$. The energy difference of spin-split dispersion laws, $\Delta E_{n}(\mathbf{k})=E_{n, s}(\mathbf{k})-E_{n,-s}(\mathbf{k})$, for s-band and heavy hole p-band are shown by dashed lines.

Band numbers $n$ and $s$ together with the wave vector k define the energy dispersion law $E_{n, s}(\mathbf{k})$ and because of the translation symmetry the velocity expectation values are given as

$$
\mathbf{v}_{n, s}(\mathbf{k})=\frac{1}{\hbar} \nabla_{\mathbf{k}} E_{n, s}(\mathbf{k}) .
$$

Since the spin operator does not commute with the Hamiltonian, expectation values of the spin-vector length $\left|\sigma_{n, s}(\mathbf{k})\right|$ can generally attain any value between 0 and 1 .

Crystalline potential can be generally expressed in the form of the Fourier series. Assuming that the local atomic potentials have the spherical symmetry, we get

$$
V(\mathbf{r})=\frac{1}{\Omega_{c}} \sum_{\mathbf{G}} \sum_{\alpha} \Omega_{\alpha} V_{\alpha}(G) e^{i \mathbf{G}\left(\mathbf{R}_{\alpha}-\mathbf{r}\right)},
$$

where $G=|\mathbf{G}|$, index $\alpha$ denotes the type of atom as well as its position $\mathbf{R}_{\alpha}$ within the unit cell of the volume $\Omega_{c}$, and $\Omega_{\alpha}$ is the atomic volume. Instead of the real crystalline potential we consider the empirical pseudopotential $V(\mathbf{r})$ representing zinc-blende structure. Fourier coefficients $V_{\alpha}(G)$ representing GaAs crystals can be found in a number of publications $[12,13]$. Note that empirical pseudopotentials do not include the contribution of low lying core states to the spin-orbit coupling term defined in Eq.(1). To get a quantitative agreement with experimentally established energy dispersions, it is necessary to add relevant correction terms $[13,14]$.

The eigenvalue problem for the above model gives energy bands showing general features corresponding to the

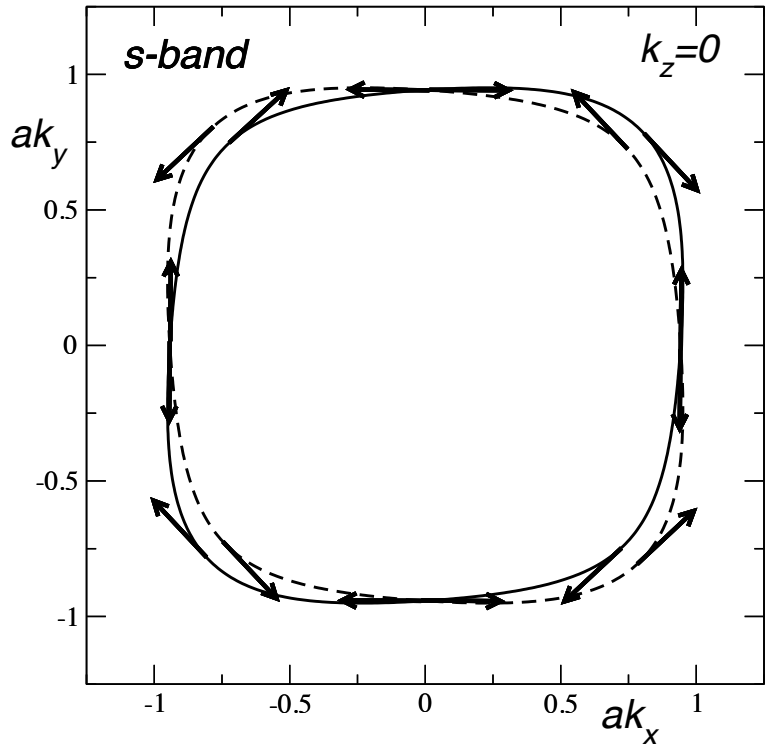

Fig. 2. Equienergy contours in the plane $(0,0,1)$ of the spin-split s-band states with $k_{z}=0$. Spin orientation is shown by arrows.

zinc-blende crystal symmetry in agreement with the Dresselhaus group analysis [2]. No spin-splitting of energy bands is found along the high symmetry directions $\Lambda$ and $\Delta$ defined by $L$ and $X$ points on the Brillouin zone boundary. Along other directions spin-splitting becomes non-zero, but it is rather weak as can be seen in Fig.1, where dispersions of s- and p-band states along $[1,1,0]$ axis and $\Delta$ direction represented by $[1,0,0]$ axis are shown.

\section{Spin polarization of Bloch states}

Spin orientation of Bloch states strongly depends on the wave vector direction. Along the directions for which spinsplitting takes place a very rich spin structure has been found. We limit our presentation of the numerical results to states at equienergy contours in the $(0,0,1)$ plane normal to the $[0,0,1]$ direction having $k_{z}=0$. In this case expectation values of $\sigma_{x}$ vanishes. Since the $[0,0,1]$ axis is a standard growth direction, these states can be considered as states representing two-dimensional systems realized in semiconductor heterostructures. For an easier presentation the equienergy contours of rather large $k$-radius are used since the tiny spin splitting increases approximately as $k^{3}$. Nevertheless, the presented properties have the same qualitative features as those obtained for smaller radii of the equienergy contours.

Equienergy contours of spin-split s-band states normal to $[0,0,1]$ crystallographic direction, 4-fold rotation axis, are shown in Fig.2 for $k_{z}=0$ together with arrows representing spin orientation of Bloch states. No spin-splitting is observed at high symmetry axes in agreement with Dresselhaus group analysis [2]. Spin is perpendicular to the velocity direction and its length $|\sigma(\mathbf{k})|$ approaches unity.

Equienergy contours for spin-split light-hole states normal to [001] crystallographic direction are shown in Fig.3 for $k_{z}=0$. Spin is nearly perpendicular to the velocity direction but its length is much less than $1,|\sigma(\mathbf{k})| \approx 0.55$.

Spin orientation of Bloch states of heavy-holes is rather rich as shown in Fig. 4. Spin is not perpendicular to the velocity and its length varies between 0 and 1 approaching 


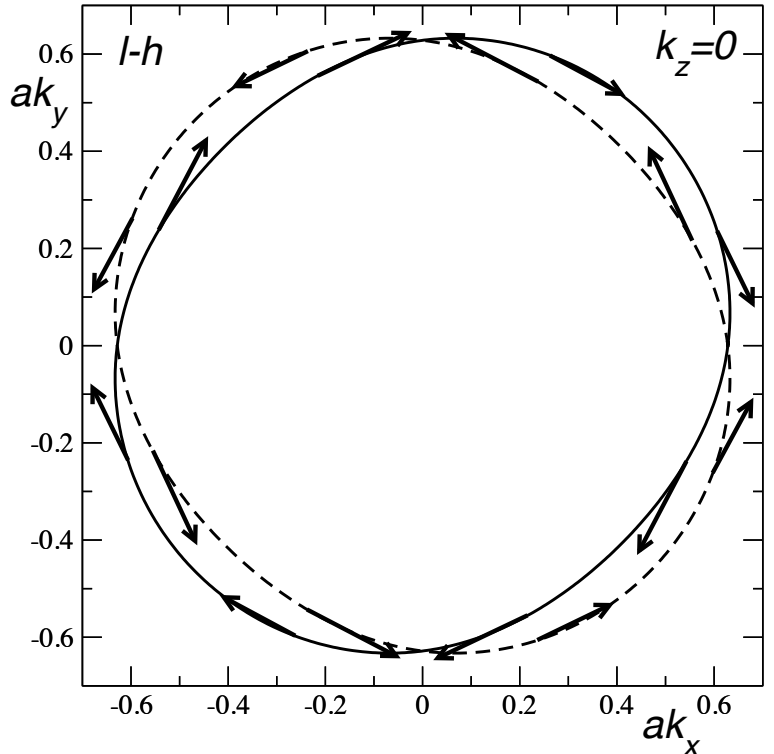

Fig. 3. Equienergy contours of the spin-split light-hole states in the plane perpendicular to the [001] direction for $k_{z}=0$. Spin orientation is shown by arrows.

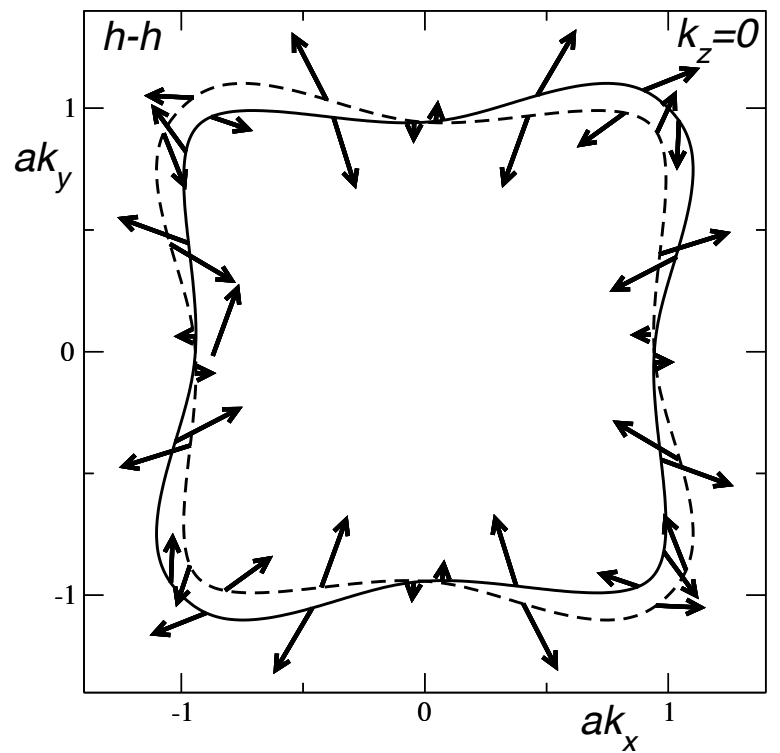

Fig. 4. Equienergy contours in the plane $(0,0,1)$ of the spin-split heavy-hole states with $k_{z}=0$. Spin orientation is shown by arrows.

zero along $\Delta$ direction, i.e., for $[ \pm 1,0,0]$ and $[0, \pm 1,0]$ crystallographic directions.

\section{Spin polarization of the Hall current densities}

Spatial distribution of the current density is defined by the spatial density of the velocity expectation values

$$
\mathbf{j}_{n, s}(\mathbf{k}, \mathbf{r})=-e \Psi_{n, s, \mathbf{k}}^{+}(\mathbf{r}) \mathbf{v} \Psi_{n, s, \mathbf{k}}(\mathbf{r}),
$$

where $\Psi_{n, s, \mathbf{k}}(\mathbf{r})$ are Bloch eigenfunctions and $\mathbf{v}$ denotes velocity operator

$$
\mathbf{v}=\frac{\mathbf{p}}{m_{0}} \sigma_{0}+\gamma \sigma \times \nabla V(\mathbf{r}) .
$$

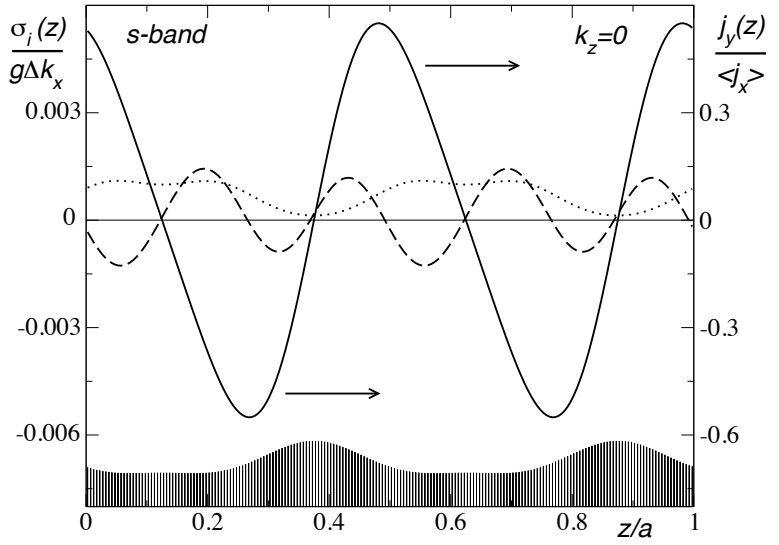

Fig. 5. Hall current density $j_{y}(z)$ (full line) and spin densities $\left(\sigma_{x}(z)\right.$ - dotted line ; $\sigma_{y}(z)$ - dashed line) for s-band electrons as functions of the $z$-coordinate representing $[0,0,1]$ crystallographic direction. The mass-density distribution of electrons at the equienergy contour is shown at the bottom of the figure.

The electric field applied, say, along the $x$-direction which is in our notation equivalent to the $[1,0,0]$ direction, induces a non-equilibrium occupation of states represented by a shift $\Delta k_{x}$ of the occupied states. In the limiting case of the zero temperature the linear response with respect to the shift $\Delta k_{x}$ gives the current density

$$
\frac{\mathbf{j}(\mu, \mathbf{r})}{\Delta k_{x}}=-\sum_{n, s} \int \delta\left(E_{n, s}(\mathbf{k})-\mu\right) \frac{\partial E_{n, s}(\mathbf{k})}{\partial k_{x}} \mathbf{j}_{n, s}(\mathbf{k}, \mathbf{r}) d \mathbf{k},
$$

where $\mu$ denotes the chemical potential. While the total current along the direction perpendicular to the applied electric field has to vanish, the corresponding local current densities are expected to oscillate through the crystal cross-section.

The contribution of a Bloch state to the local spin density reads

$$
\sigma_{n, s}(\mathbf{k}, \mathbf{r})=\Psi_{n, s, \mathbf{k}}^{+}(\mathbf{r}) \sigma \Psi_{n, s, \mathbf{k}}(\mathbf{r}) .
$$

As a consequence of the Kramer's theorem, the sum of contributions of states at equienergy contours vanishes. Non-equilibrium ocuppation of states induced by the applied electric field gives rise to a non-zero local spin density. In analogy with the procedure presented above for the current density, local spin polarizations can be established.

As an example let us consider a two-dimensional gas of carriers represented by bulk states with $k_{z}=0$. For this case the Hall current density averaged over $x$ - and $y$ coordinates will be established giving its distribution along the $\hat{z}$ direction

$$
j_{y}(z)=\int \mathbf{j}(\mu, \mathbf{r}) d x d y .
$$

Spin polarization of the Hall current density $j_{y}(z)$ is thus defined by the corresponding spin polarization averaged over $x$ - and $y$ - coordinates, $\sigma_{x}(z)$ and $\sigma_{y}(z)$. Note that for $k_{z}=0$ the only spin components normal to $\hat{z}$ direction can be induced.

Typical results for s-band electrons are presented in Fig. 5, where the $z$-dependence of the Hall current density $j_{y}(z)$ normalised to the averaged applied current density $\left\langle j_{x}\right\rangle$ together with spin densities $\sigma_{x}(z)$ and $\sigma_{y}(z)$ nor- 


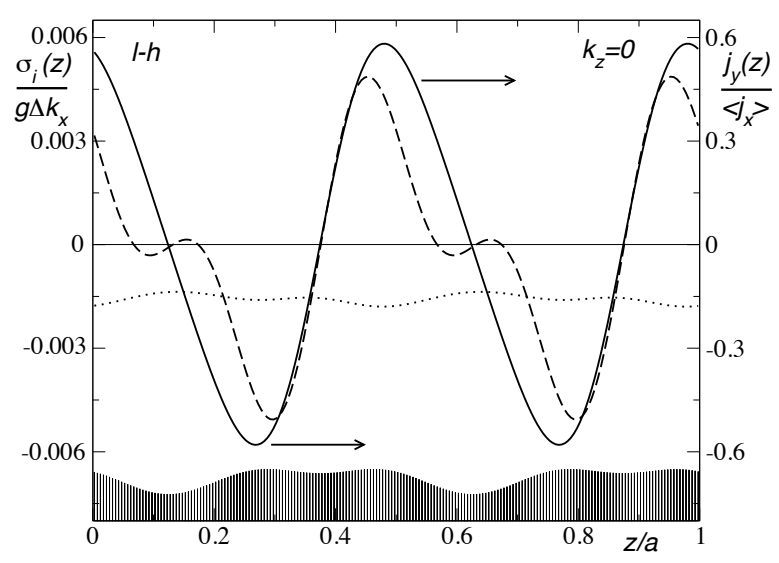

Fig. 6. Hall current density $j_{y}(z)$ (full line) and spin densities $\left(\sigma_{x}(z)\right.$ - dotted line ; $\sigma_{y}(z)$ - dashed line) for light holes as functions of the $z$-coordinate representing $[0,0,1]$ crystallographic direction. The mass-density distribution of electrons at the equienergy contour is shown at the bottom of the figure.

malised to the product of the equienergy density of states and $k_{x}$ shift, $g \Delta k_{x}$, are shown.

We used the same data as that giving for the energy $\mu$ the equienergy contours shown in Fig. 2. Oscillations of the Hall current density are caused by the non-zero orbital momentum of Bloch states having different mass-centre positions for opposite wave vectors $\mathbf{k}$. As expected, the total Hall current as well as the total $\sigma_{y}$ spin-component vanish. On the other hand, the non-vanishing total $\sigma_{x}$ spincomponent indicates the already described spin polarization of the system induced by the applied current [3-5]. No total spin transfer perpendicular to the applied current, spin Hall current, has been observed.

A stronger spin polarization of the Hall current densities could be expected for p-band states having larger orbital moments than s-band states. Especially for light holes showing substantially larger spin splitting, the pronounced effect is expected despite the fact that the length of the spin vector is smaller, $|\sigma(\mathbf{k})| \approx 0.55$. The results obtained by using the same data as that giving the equienergy contours shown in Fig. 3 are presented in Fig. 6. As in the preceding case, the total Hall current as well as the total $\sigma_{y}$ spin-component vanish. On the other hand, the tendency to transfer spins with different sign of their $y$-component in opposite directions, spin Hall current, is clearly seen.

Note that for both presented examples the contribution of the spin-orbit coupling term entering velocity operator, Eq.(9), gives only small corrections.

We have presented examples for chemical potentials that give equipotential contours with quite large average radii $\left\langle k_{c}\right\rangle,\left\langle k_{c}\right\rangle a \sim 1$, which corresponds to unrealistic carrier concentrations. With decreasing values of the $\left\langle k_{c}\right\rangle$, the ratio of the Hall current density and the applied current decreases similarly as spin splitting, i.e., as $\left\langle k_{c}\right\rangle^{3}$. Similarly, the corresponding spin polarization is suppressed. Nevertheless, up to values $\left\langle k_{c}\right\rangle a \sim 0.3$ the qualitative features remain unchanged. For smaller radii the accuracy of our numerical procedures especially for the s-band electrons and heavy holes becomes unsatisfactory because of the very weak spin splitting of equienergy lines.

Non-zero spin Hall current has also been indicated for heavy holes. The oscillation amplitudes of the Hall current density and the spin density along $\hat{z}$ direction are less but of the same order as that for light holes. However, opposite to the s-state electrons and light holes the observed properties are not so universal. The reason is that their spin structure as well as the shape of equienergy lines depend on the heavy hole concentration. Note that there even appears crossing of the energy dispersions as indicated in Fig. 1 by the arrow.

Let us note that in the three-dimensional case the spin polarization becomes more complex because of the nonzero contribution of the spin $z$-component and non-zero Hall current densities along $z$-direction.

\section{Concluding remarks}

We have studied the spin polarization of Bloch states in semiconductors with zinc-blende structure using the empirical pseudopotential method. Knowledge of Bloch eigenfunctions allowed us to analyse real-space spin and current distributions. In the current carrying regime it results in the local spin polarization accompanied by spin-polarized Hall-current densities. We have shown that at least in the case of light holes they are responsible for transferring spins of different orientation in opposite directions. It results in the spin Hall current expressed by quantities measurable at least in principle.

Nevertheless, for full understanding of spin currents the presented analysis needs a modification allowing to take into account the effect of the sample edges together with realistic conditions for potential well boundaries. Particularly it is important in the case of the spin accumulation at the sample edges [15], which is up to now the only observable manifestation of the spin Hall effect.

\section{References}

1. J. M. Luttinger, Phys. Rev. 102, (1956) 1030.

2. G. Dresselhaus, Phys. Rev. 100, (1955) 580.

3. E. L. Ivchenko, and G. E. Pikus, Pisma Zh. Eksp. Teor. Fiz. 27, (1978) 640 [JETP Lett. 27, (1978) 604].

4. V. M. Edelstein, Solid State Comm. 73, (1990) 233.

5. A. G. Aronov, Yu. B. Lyanda-Geller, and G. E. Pikus, Zh. Eksp. Teor. Fiz. 100, (1991) 973 [Sov. Phys. JETP 73, (1991) 537].

6. S. Zhang, Phys. Rev. Lett. 85, (2000) 393.

7. J. Sinova, S. Murakami, S.-Q. Shen, and M.-S. Choi, Solid State Commun. 138, (2002) 214.

8. J. Sinova, D. Culcer, Q. Niu, N. A. Sinitsyn, T. Jungwirth, and A. MacDonald, Phys. Rev. Lett. 92, (2004) 126603.

9. G. Y. Guo, Yugui Yao, and Qian Niu, Phys. Rev. Lett. 94, (2005) 226601.

10. J. Shi, P. Zhang, D. Xiao, and Q. Niu, Phys. Rev. Lett. 96, (2006) 076604.

11. E. I. Rashba, Phys. Rev. B 68, (2003) 241315(R).

12. M. L. Cohen and T. K. Bergstresser, Phys. Rev. 141, (1966) 789.

13. K. A. Mäder and A. Zunger, Phys. Rev. B 50, (1994) 17393.

14. S. Bloom and T. K. Bergstresser, Solid State Commun. 6, (1970) 465.

15. T. D. Stanescu, and V. Galitski, Phys. Rev. B 74, (2006) 205331. 